\title{
A Compact Ultrathin Ultra-wideband Metamaterial Microwave Absorber
}

\author{
D. Sood*, Chandra C. Tripathi, \\ Department of Electronics \& Communication Engineering, University Institute of Engineering \& Technology, \\ Kurukshetra University, Kurukshetra \\ *Email: deepaksood.uiet@gmail.com
}

\begin{abstract}
A simple design of compact ultra-wideband, ultra-thin metamaterial microwave absorber has been presented. The unit cell of the proposed design is composed of diagonally placed 'microstrip bend' like patches printed on grounded dielectric substrate. The simulation results demonstrate the $10-\mathrm{dB}$ absorption bandwidth of 7.19 GHz from 10.45 GHz to $17.64 \mathrm{GHz}$ and FWHM (full width at half maximum) bandwidth of $11.43 \mathrm{GHz}$ from $9.61 \mathrm{GHz}$ to 21.04 GHz. The proposed design presents more than $90 \%$ absorption for a bandwidth of $1.02 \mathrm{GHz}$ at wide angle of incidence up to $60^{\circ}$. The absorption mechanism has been analyzed through surface current and electromagnetic field distributions. The proposed design has been fabricated and experimentally tested for its 10-dB absorption bandwidth under different polarization and oblique angles of incidence of electromagnetic wave. The designed absorber is low profile (unit cell size is $\sim 0.257 \lambda_{0}$ at the center frequency corresponding to 10-dB absorption bandwidth), ultrathin $\left(\sim \lambda_{0} / 14\right.$ thick at the center frequency) and provides a novel design of wideband absorber for many useful applications.
\end{abstract}

Index Terms - Broadband absorber, metamaterial, low profile design, wideangle absorber, monolayer microwave absorber.

\section{INTRODUCTION}

The artificially engineered electromagnetic metamaterials have inspired the researchers due to their unusual, exotic properties of simultaneous negative permittivity $(\varepsilon)$ and permeability $(\mu)[1]$. They have found tremendous applications which spans from microwave to optical frequency regime [2], such as RCS reduction [3], radar imaging [4], solar cells [5], photo-detectors [6], chip less RFID [7], cloaking [8], antennas [9], perfect lens [10] and phase modulators [11]. Nearly perfect absorption, ultrathin thickness, ease of fabrication and increasing effectiveness makes the metamaterial absorbers more popular than the conventional electromagnetic absorbers. The incident electromagnetic fields on a metamaterial absorber manipulate the effective permittivity and permeability of the structure in such a way that its input impedance becomes equal to the free space impedance. This minimizes the reflection from the structure, thereby causing the absorption of the incident wave. In spite of the nearly perfect absorption with ultrathin thickness, metamaterial based absorbers have narrow operational bandwidth due to their resonant behaviour [12]. The first reports on multiband absorber $[13,14]$, set the basis to multiband and broadband absorbers. Till date, few monolayer layer designs with a small enhancement in bandwidth have been proposed $[15,16]$. However, it is cumbersome to 
tune each resonant frequency at one time, in such complicated multi-resonant structures. Some multilayer designs with enhanced bandwidth have been presented [19-22]. Although, they exhibit wideband absorption response, but the large thickness and complexity involved in the alignment of layers makes them unsuitable for conformal planar applications. Another technique of bandwidth enhancement is to combine different resonant structures to form an array of differently scaled structures as presented in $[21,22]$. However, the large unit cell dimension is a prime limiting factor in such designs to be practically viable. Use of passive lumped element in ELC resonator based absorbers is another approach for bandwidth enhancement [23]. Although, this technique increases the bandwidth but it requires an additional fabrication process, which can cause degradation in absorber performance. An ultrawideband ultrathin metamaterial absorber is presented in [24] that provide 10$\mathrm{dB}$ absorption bandwidth of $4.4 \mathrm{GHz}(43 \%$ at $10 \mathrm{GHz})$. However, it does not provide wide angle absorptivity. Another triple layer broadband metamaterial absorber based on fractal tree structure has been presented in [25]. Although it provides wide angle absorptivity but its thickness and unit cell size is quite large. A wideband low profile, ultrathin metamaterial absorber has presented in [26]. Despite of its ultrathin thickness, low profile design and wideband response, the $10 \mathrm{~dB}$ absorption bandwidth is only $35.8 \%$.

In this paper, a novel ultra-wideband low profile wide-angle ultrathin metamaterial absorber has been presented. The proposed absorber exhibits more than $90 \%$ absorption of $7.19 \mathrm{GHz}$ from 10.45 to $17.64 \mathrm{GHz}$ and FWHM (full width at half maximum) bandwidth of $11.43 \mathrm{GHz}$ from 9.61 to 21.04 GHz. Two microstrip bend like patches are placed diagonally, whose geometrical dimensions are optimized in such a way that absorption frequencies come closer and give wideband absorption response. The simulated result shows more than $90 \%$ absorption bandwidth of $1.02 \mathrm{GHz}$ from 11.27 to $12.29 \mathrm{GHz}$ for wide incident angle of 600 . The impedance characteristics, electromagnetic field and surface current distributions have been analyzed to understand the physical mechanism of absorption in the proposed absorber. The designed structure has been studied for different incidence angles under TE polarization and for different polarization angles under normal incidence of electromagnetic wave. Moreover, essential design parameters are varied in order to analyze their contribution in wideband absorption. A prototype array of the proposed absorber has been fabricated using standard PCB technology and experimentally tested for its above $90 \%$ absorption bandwidth. The experimental results are observed in agreement with the simulated responses. In comparison to the already presented metamaterial absorbers, the proposed structure is a wideband low profile absorber with ultrathin thickness and provides wide-angle response and therefore it is suitable for many potential applications.

\section{DESIGN AND SimULATION}

In Fig. 1(a) the unit cell design of the proposed ultra-wideband absorber is shown. It consists of top and bottom metallic layers separated by dielectric substrate FR-4 (permittivity $\varepsilon_{\mathrm{r}}=4.4$ and loss 
tangent $=0.02$ ). The top layer consists of diagonally placed, two 'microstrip bend' like patches. The overall size of the unit cell is just $5.5 \mathrm{~mm}$, which is $0.257 \lambda_{0}$ at the center frequency of $14.05 \mathrm{GHz}$ corresponding to $-10 \mathrm{~dB}$ absorption bandwidth. The substrate used is of thickness $1.6 \mathrm{~mm}$ which is $\lambda_{0} / 14$ at the center frequency. The top and ground layers are of copper $\left(\sigma=5.8 \times 10^{7} \mathrm{~S} / \mathrm{m}\right)$ with a thickness of $3.5 \mu \mathrm{m}$. The optimized dimensions of the unit cell as shown in Fig. $1($ a $)$ are: $\mathrm{L}=3.5 \mathrm{~mm}$, $\mathrm{W}=1.4 \mathrm{~mm}, \mathrm{~L}_{1}=1.7 \mathrm{~mm}, \mathrm{~S}_{1}=2.54 \mathrm{~mm}$ and $\mathrm{S}_{2}=0.56 \mathrm{~mm}$. The absorptivity of the proposed absorber is calculated as: $A=1-\left|S_{11}\right|^{2}-\left|S_{21}\right|^{2}$. Here ' $A$ ' is the absorptivity, $\left|S_{11}\right|^{2}$ is the reflected power and $\left|S_{21}\right|^{2}$ is the transmitted power. As there is no transmitted power due to metallic ground layer so $\left|S_{21}\right|^{2}=0$ and the absorptivity is $A=1-\left|S_{11}\right|^{2}$.

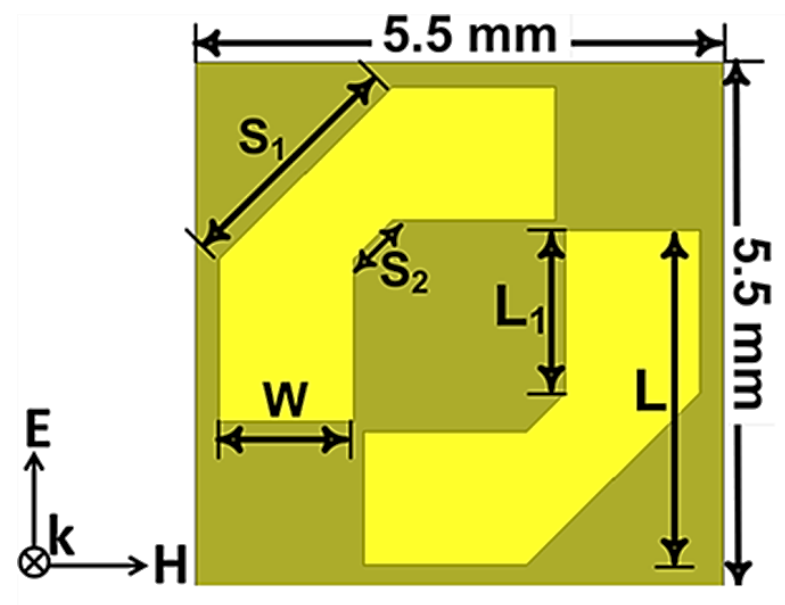

(a)

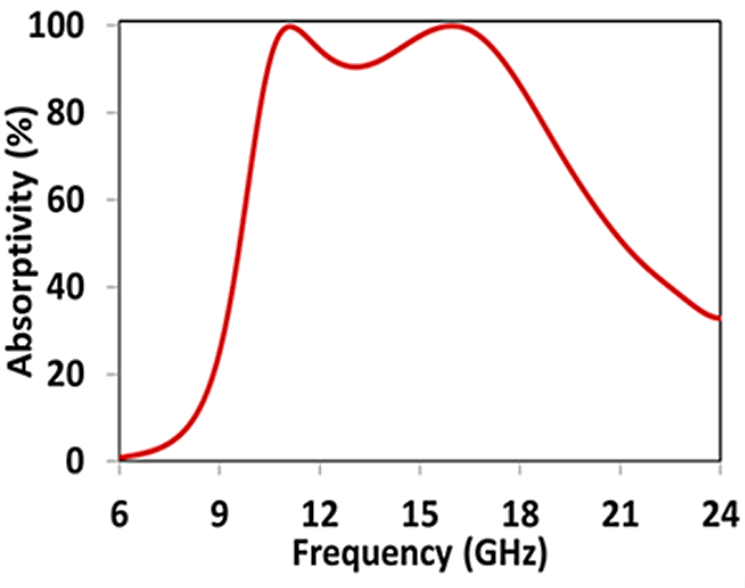

(b)

Fig. 1. (a) Front view of the unit cell structure and (b) simulated absorptivity under normal incidence.

For the analysis of the infinite array of the proposed absorber, the unit cell is simulated with Floquet's periodic boundary conditions using ANSYS HFSS. The simulated result for the normal incidence of the electromagnetic wave is shown in Fig. 1(b). It is observed that the absorption is more than $90 \%$ from 10.45 to $17.64 \mathrm{GHz}$, which is $51.2 \%$ at the center frequency of $14.05 \mathrm{GHz}$. The two absorption peaks $\left(f_{1} \& f_{2}\right)$ are observed at 11.13 and $15.99 \mathrm{GHz}$ with absorptivities of $99.78 \%$ and $99.96 \%$ respectively. It is also seen that the FWHM absorption bandwidth of the proposed absorber is 11.43 GHz, which covers almost $69 \%$ of $\mathrm{X}$ - band, $100 \%$ of $\mathrm{Ku}-$ band and $36 \%$ of $\mathrm{K}$-band. The normalized input impedance $(\mathrm{Z})$ plot for the designed absorber is shown in Fig. 2. At both the absorption peaks the real parts of the impedance $(Z)$ are close to unity and imaginary parts are close to zero. This implies that at both the absorption peaks normalized input impedance matches closely to free space impedance $\mathrm{Z}_{0}$ which support absorption phenomenon.

In order to study the polarization behavior of the proposed metamaterial absorber the structure is analyzed under different polarization angles $(\phi)$ from $0^{0}$ to $90^{\circ}$ as shown in Fig. 3. Under this condition the direction of wave propagation is along z-axis and electric and magnetic field vector makes an angle ' $\phi$ ' with $\mathrm{x}$ axis and $\mathrm{y}$ axis respectively. It is observed that the absorptivity decreases 
to minimum as polarization angle reaches $45^{\circ}$ and then it again increases to a maximum as polarization angle increases to $90^{\circ}$. From this simulated response, it is clear that the proposed structure is two-fold symmetric in design. The proposed absorber has been studied for different oblique incidence angles $(\theta)$ under TE polarization as shown in Fig. $4(a)$. In this situation electric field is along $\mathrm{X}$-axis but magnetic field and direction of wave vector are changed with an angle ' $\theta$ ' w.r.t ' $\mathrm{Y}$ ' and ' $\mathrm{Z}$ '-axis respectively. It is observed that the proposed absorber provides high absorption with wide absorption bandwidth up to large incidence angles. It is seen that at the incident angle of $40^{\circ}$ the absorptivity is more than $70 \%$ from 10.35 to $20.68 \mathrm{GHz}$. Moreover, at the incident angle of $60^{\circ}$, the proposed absorber provides more than $50 \%$ absorption with the bandwidths of $5.09 \mathrm{GHz}$ from 10.37 to $15.46 \mathrm{GHz}$ and $7.01 \mathrm{GHz}$ from 15.77 to $22.78 \mathrm{GHz}$. Further, as the incident angle reaches to $80^{\circ}$, the second absorption peak disappears and absorptivity is more than $60 \%$ from 11.71 to $13.04 \mathrm{GHz}$.

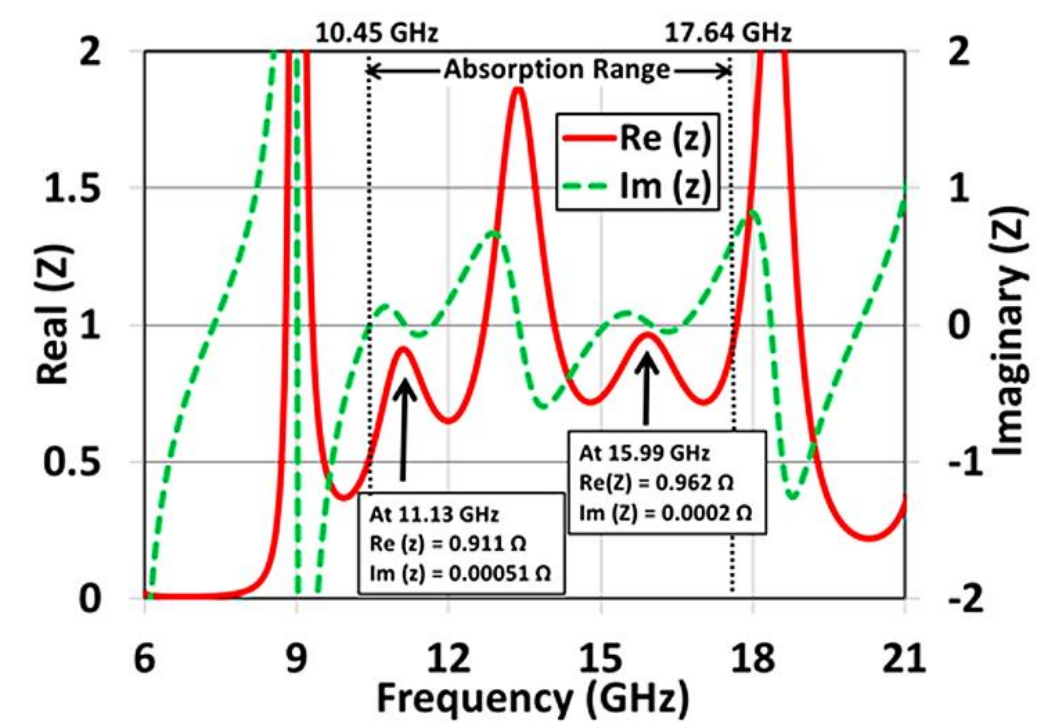

Fig. 2. Normalized input impedance plot of the proposed absorber structure.

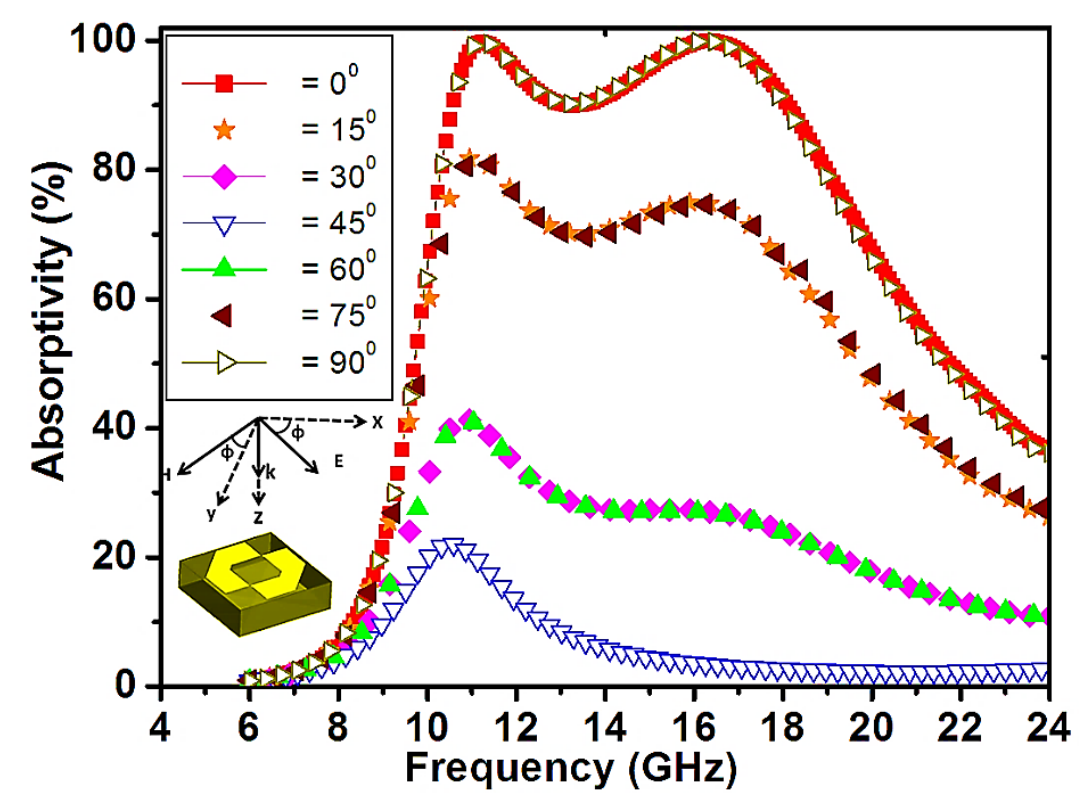


Fig. 3. Simulated absorptivity of the proposed absorber for various polarization angles $(\varphi)$ under normal incidence.

Further, the performance of the absorber is also studied for different oblique incident angles under TM polarization. The simulated response is shown in Fig. 4(b). In this case magnetic field vector is along y-axis while electric field vector and wave vector are varying by an angle ' $\theta$ '. As the design is two-fold symmetric therefore, similar to the case of TE polarization, the wide absorption bandwidth up to large incidence angles is also observed for TM polarization as per the similar observations reported in $[27,28]$. At the incident angle of $40^{\circ}$ the absorptivity is more than $70 \%$ from 10.34 to $20.51 \mathrm{GHz}$. Further, at the incident angle of $60^{\circ}$, absorption is more than $50 \%$ with the bandwidths of 5.01 GHz from 10.33 to $15.34 \mathrm{GHz}$ and $6.97 \mathrm{GHz}$ from 15.70 to $22.67 \mathrm{GHz}$.

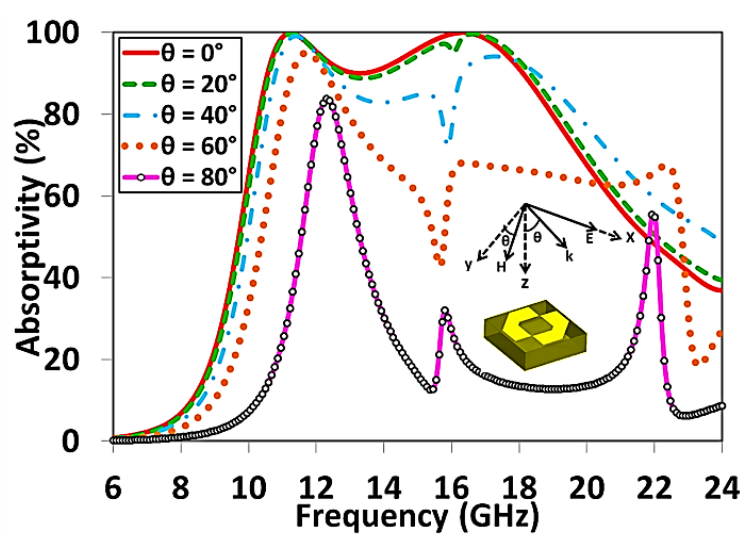

(a)

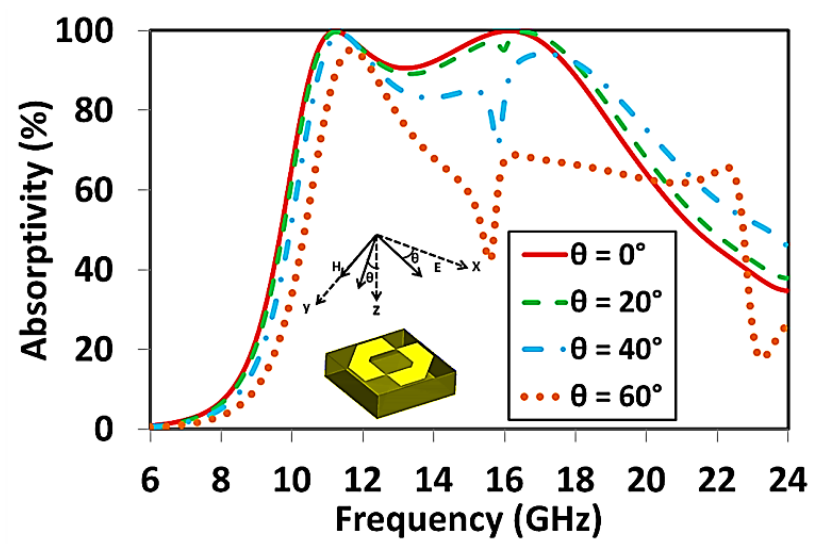

(b)

Fig. 4. (a) Simulated absorptivity under different oblique incidence angles (' $\theta$ ') for (a) TE and (b) TM polarizations.

\section{(A) Physical Mechanism of Absorption}

In order to better understand the absorption mechanism in the proposed ultra-wideband absorber, the electric and magnetic field distributions have been studied at both the absorption peaks i.e. $f_{1}$ and $f_{2}$ as shown in Fig. 5. It is observed from Fig. $5(a)$ that at the lower absorption peak $\left(f_{l}\right)$ of $11.13 \mathrm{GHz}$, the lateral outer edges (having dimension 'L1') and the width ('W') of the metallic patches are the main contributor of providing high electric field around the structure. On the other hand it is seen from Fig. 5 (b) that the magnetic field is mainly distributed in the dielectric region of the proposed design, which clearly proves that the high dielectric loss is responsible for this strong magnetic field in the structure. It is observed from the Fig.5 (c) that at the higher absorption frequency $\left(f_{2}\right)$ of $15.99 \mathrm{GHz}$, the small gap at the corners of the two diagonally placed 'microstrip bend' like patches and the outer slanting edges (having dimension ' $\mathrm{S}_{1}$ ') are the primary contributor of providing high electric field in the structure. Although, the inner slating edges (having dimension ' $\mathrm{S}_{2}$ ') provide high magnetic field in the structure, but the magnetic field is also distributed in the dielectric region at this higher absorption peak as shown in Fig. $5(d)$. The surface current distributions for the two absorption peaks at the top and bottom surfaces have also been shown in Fig. 6. It is clearly observed that at the lower absorption 
peak $\left(f_{l}\right)$ of $11.13 \mathrm{GHz}$ the surface currents are uniformly distributed over the top metallic patches, but at the higher absorption peak $\left(f_{2}\right)$ of $15.99 \mathrm{GHz}$ the surface current is mainly distributed at the outer (' $\mathrm{S}_{1}$ ') and inner (' $\mathrm{S}_{2}$ ') slanting edges of the design.

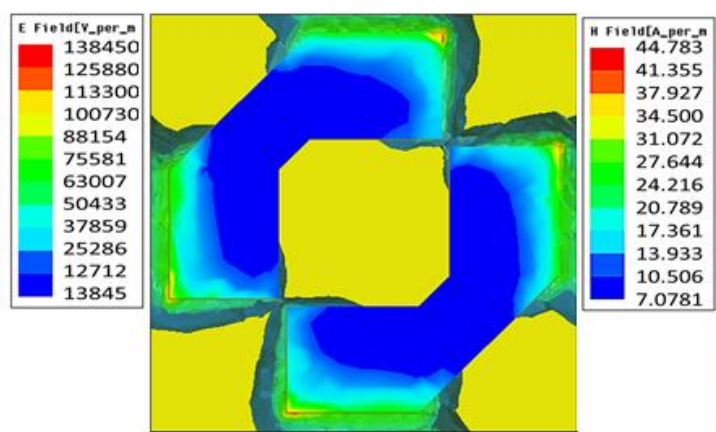

(a)

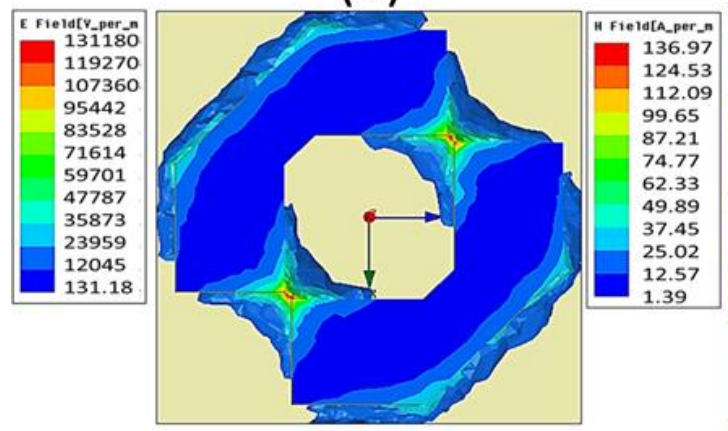

(c)

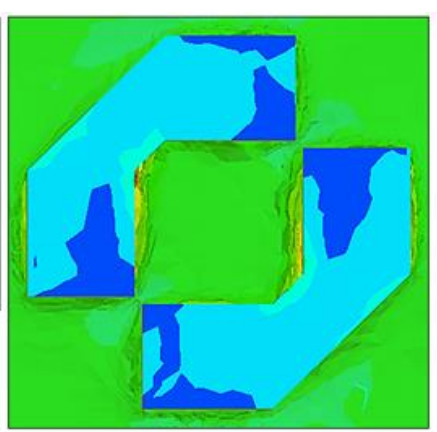

(b)

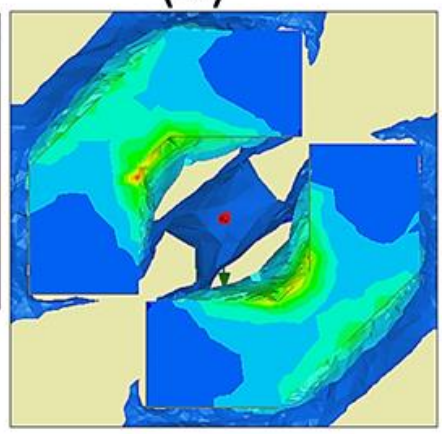

(d)

Fig. 5. Simulated field distribution at $11.13 \mathrm{GHz}$ (a) electric field (b) magnetic field and at $15.99 \mathrm{GHz}$ (c) electric field (d) magnetic field.

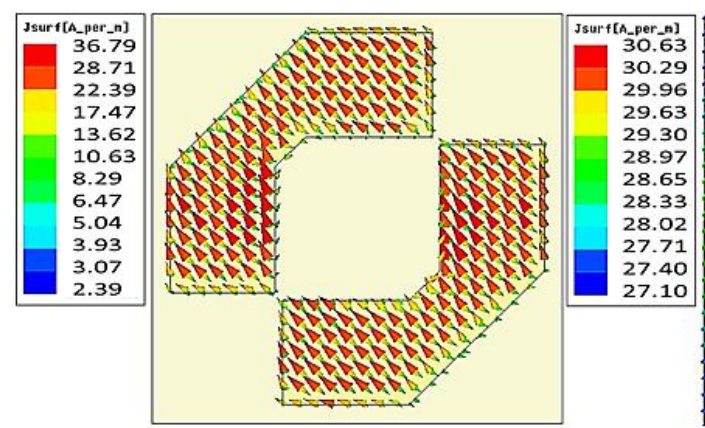

(a)

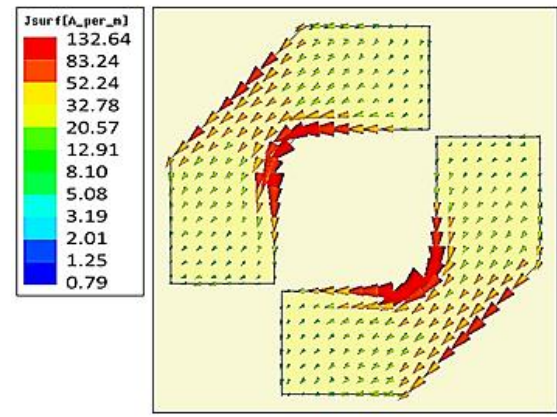

(c)

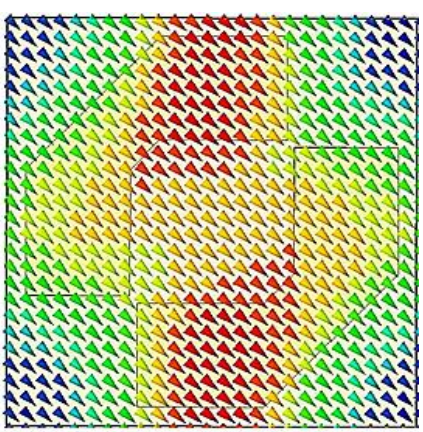

(b)

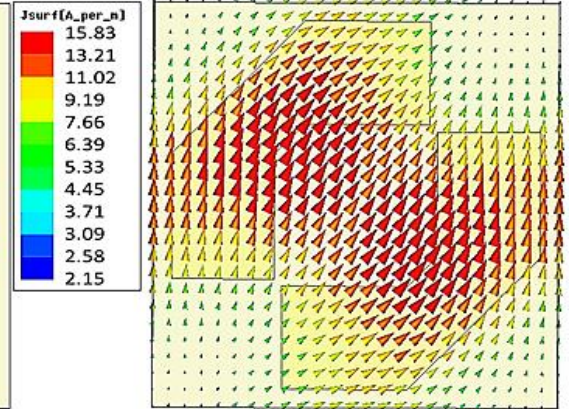

(d) 
Fig. 6. Surface current distribution at $11.13 \mathrm{GHz}$ (a) top surface (b) bottom surface and at $15.99 \mathrm{GHz}$ (c) top surface (d) bottom surface.

However, at both the absorption peaks the surface currents are antiparallel on the top and bottom surfaces forming a circulating loop around the incident magnetic field and create a magnetic coupling. The incident electric field is coupled with the diagonally placed top metallic patches. At the absorption frequencies, these strong electric and magnetic resonances are responsible for realizing the high absorption in the proposed design.

\section{(B) Contribution of dimensional parameters in absorption}

Different approaches have been employed to provide better insight of the physics behind the dependence of absorption mechanism on the geometric parameters of the metamaterial based absorber structures [29-32]. Efforts also been done to derive suitable formulas with equivalent circuits based on transmission line theory [29], lumped circuit theory [30] and coupled line theory [31]. But these techniques are still limited to simple geometries such as metallic wire and square loop. However, the location of absorption frequencies can be controlled by tailoring the effective inductance and capacitance through changing the current paths and periodicity respectively of the unit cell of the absorber structures [32]. Both the bandwidth and absorptivity can be controlled by tailoring all the geometric dimensions of such sub-wavelength metamaterial absorber structures. Therefore, in order to gain better insight about the contribution of various dimensional parameters in the absorption bandwidth, the essential dimensions of the proposed design has been varied over their range of extension. The width ('W') of the proposed design contributes highly in the absorption bandwidth. It has been varied from $1.0 \mathrm{~mm}$ (where dual band has been noticed) to $1.4 \mathrm{~mm}$ (maximum limit as per design) as shown in Fig. 7 (a). It is observed that the variation in width mainly affects the higher absorption peak $\left(f_{2}\right)$. Initially, for ' $\mathrm{W}$ ' equals to $1.0 \mathrm{~mm}$ two absorption peaks are observed at 10.41 and $18.78 \mathrm{GHz}$, with a bandwidth of $15.17 \mathrm{GHz}(9.44$ to $24.61 \mathrm{GHz})$ with more than $60 \%$ absorptivity. As the width ('W') increases to $1.2 \mathrm{~mm}$, the absorption peaks are at 10.86 and 17.43 $\mathrm{GHz}$ and above $80 \%$ absorptivity is noticed from 10.02 to $20.27 \mathrm{GHz}$. For the further increase in 'W' to $1.4 \mathrm{~mm}$ the absorptivity is above $90 \%$ from 10.45 to $17.64 \mathrm{GHz}$ and the absorption peaks are observed at 11.13 and $15.99 \mathrm{GHz}$.

Another parameter which affects the absorption bandwidth is the length ' $\mathrm{L}_{1}$ '. It has been varied from $0.8 \mathrm{~mm}(\mathrm{~min})$ to $1.7 \mathrm{~mm}(\max )$ as shown in Fig. $7(b)$. It is observed that at the optimized value ' $\mathrm{L}_{1}$ ' i.e. $1.7 \mathrm{~mm}$, two absorption peaks are observed at 11.13 and $15.99 \mathrm{GHz}$ with more than $90 \%$ absorption bandwidth of $7.19 \mathrm{GHz}$. However, at ' $\mathrm{L}_{1}$ ' equals to $0.8 \mathrm{~mm}$ the two absorption peaks observed to be merged with more than $90 \%$ absorption bandwidth of only $4.38 \mathrm{GHz}$ from 15.51 to 19.89 GHz. Since the increase in length, increases the effective inductance thereby causes the resonance frequencies to decrease, therefore in the proposed design both the absorption peaks shifts to lower values with the gradual increase in length ' $\mathrm{L}_{1}$ '. Moreover, it is also observed that the variation 
in ' $\mathrm{L}_{1}$ ' largely affects the lower absorption peak $\left(f_{l}\right)$. The variation in absorption peaks w.r.t the variation in ' $\mathrm{L}_{1}$ ' is shown in Table I. Although, at ' $\mathrm{L}_{1}$ ' equals to $1.4 \mathrm{~mm}$ the absorptivity is above $90 \%$ with an absorption bandwidth of $7.79 \mathrm{GHz}$ from 11.65 to $19.44 \mathrm{GHz}$, which is slightly more than for its optimized value of $1.7 \mathrm{~mm}$ but this optimized value of ' $\mathrm{L}_{1}$ ' is chosen on the basis of the frequency range of the Horn antennas (1 to $18 \mathrm{GHz}$ ) used for measurements.

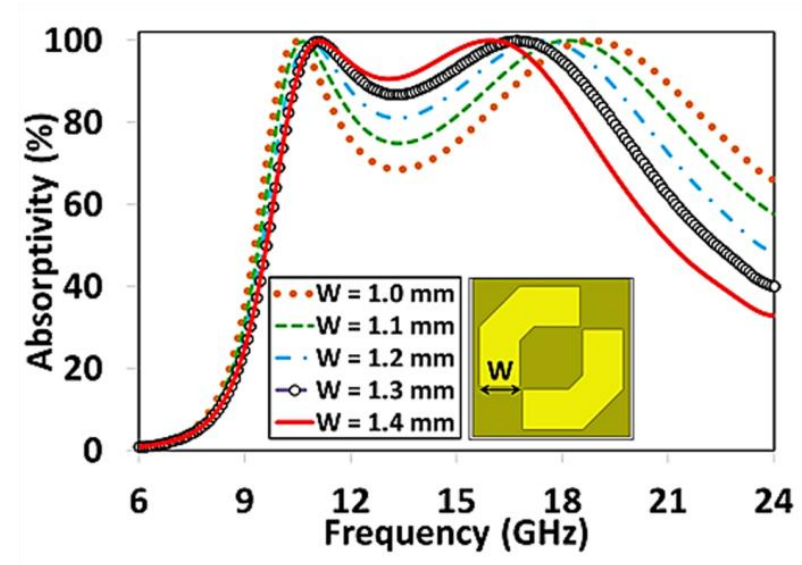

(a)

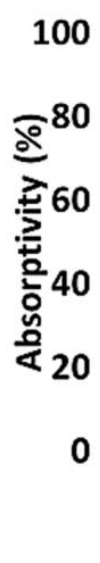

6

(b)

Fig. 7. Simulated absorptivity (a) for different widths ('W') and (b) for different length (' $L_{1}$ ') of the proposed absorber.

TABLE I. VARIATIONS IN ABSORPTION FREQUENCIES W.R.T VARIATION IN LENGTH 'L1'.

\begin{tabular}{|c|c|c|c|c|}
\hline S. No. & $\mathbf{L}_{\mathbf{1}}(\mathbf{m m})$ & $f_{\boldsymbol{l}}(\mathbf{G H z})$ & $\boldsymbol{f}_{\mathbf{2}} \mathbf{( G H z )}$ & $\begin{array}{c}\text { 10 dB Absorption } \\
\text { Bandwidth (GHz) }\end{array}$ \\
\hline 1 & 0.8 & - & - & 4.38 \\
\hline 2 & 1.1 & 14.46 & 17.43 & 7.11 \\
\hline 3 & 1.4 & 12.48 & 17.25 & 7.79 \\
\hline 4 & 1.7 & 11.13 & 15.99 & 7.19 \\
\hline 5 & $\begin{array}{c}\text { \% Change in } \\
\text { absorption peak } \\
\text { w.r.t change in } \mathrm{L}_{1}\end{array}$ & $23.02 \%$ & $8.26 \%$ & - \\
\hline
\end{tabular}

Other important design parameters to study are the outer (' $\mathrm{S}_{1}$ ') and inner (' $\mathrm{S}_{2}$ ') slanting edges in the proposed design. In order to understand the contribution of these slanting edges in the absorption bandwidth, the absorber structure has been analyzed under three different configurations as shown in Fig. 8 (a). At first, in configuration 'I' the structure is analyzed without slanting edges i.e. only two ' $L$ ' shaped diagonally placed patches are studied. It is observed that the absorption bandwidth is above $90 \%$ for only $3.26 \mathrm{GHz}$ from 10.65 to $13.91 \mathrm{GHz}$. In the 'II' configuration, slanting edges are created at one ' $L$ ' shaped patch only and it is seen that the above $90 \%$ absorption bandwidth increases to $4.97 \mathrm{GHz}$ from 10.55 to $15.52 \mathrm{GHz}$. Finally, in configuration 'III' i.e. the proposed design in which slating edges are created at both the patches it is observed that the $90 \%$ absorption bandwidth increases to $7.19 \mathrm{GHz}$. Further, to investigate the effects of slanting edges on the absorption peaks, the dimensions of ' $\mathrm{S}_{1}$ ' and ' $\mathrm{S}_{2}$ ' are varied as shown in Fig. $8(b)$ and $(c)$ respectively. As the decrease in 
inductance increases the absorption frequency, therefore in order to get the absorption at the same frequency the periodicity of the absorber structure is required to be enhanced to increase the effective capacitance as reported [32]. Thus, to design a compact absorber structure with the fixed periodicity, the absorption frequency can be controlled by varying the effective inductance of the structure with the help of tailoring its geometric dimensions and controlling the electromagnetic field coupling between the resonant elements present in the unit cell. It is observed from Fig. $8(b)$ that with the increase in ' $\mathrm{S}_{1}$ ' while keeping ' $\mathrm{S}_{2}$ ' fixed the lower absorption peak $\left(f_{l}\right)$ remains almost unchanged, whereas the higher absorption peak $\left(f_{2}\right)$ starts increasing as effective inductance of the structure gets lowered due to smaller path travelled by currents at the outer edges. This results the increase in absorption bandwidth. Initially, at ' $\mathrm{S}_{1}$ ' equals to $1.4 \mathrm{~mm}$ the two absorption peaks are observed at 11.47 and $13.74 \mathrm{GHz}$ with $90 \%$ absorption bandwidth of $4.75 \mathrm{GHz}$ from 10.56 to $15.31 \mathrm{GHz}$.

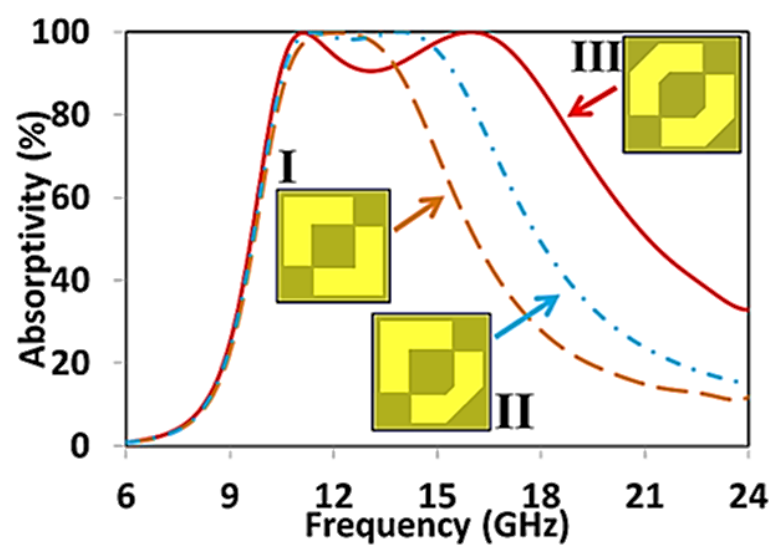

(a)

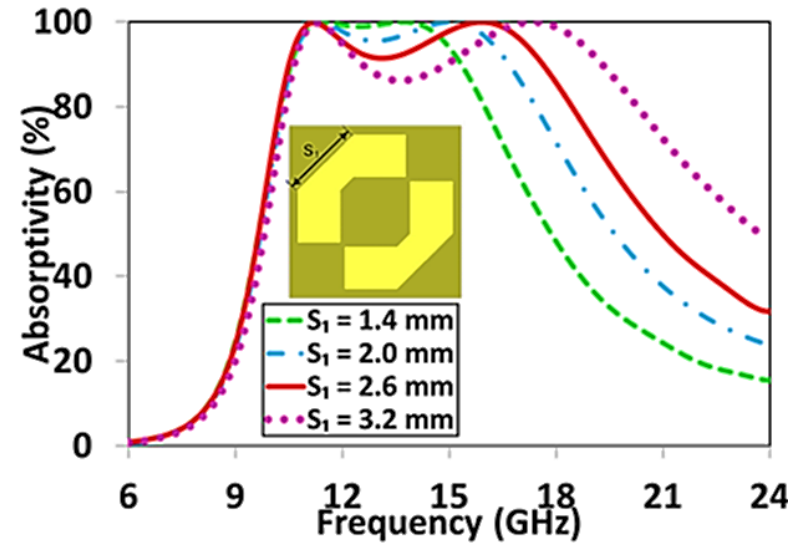

(b)

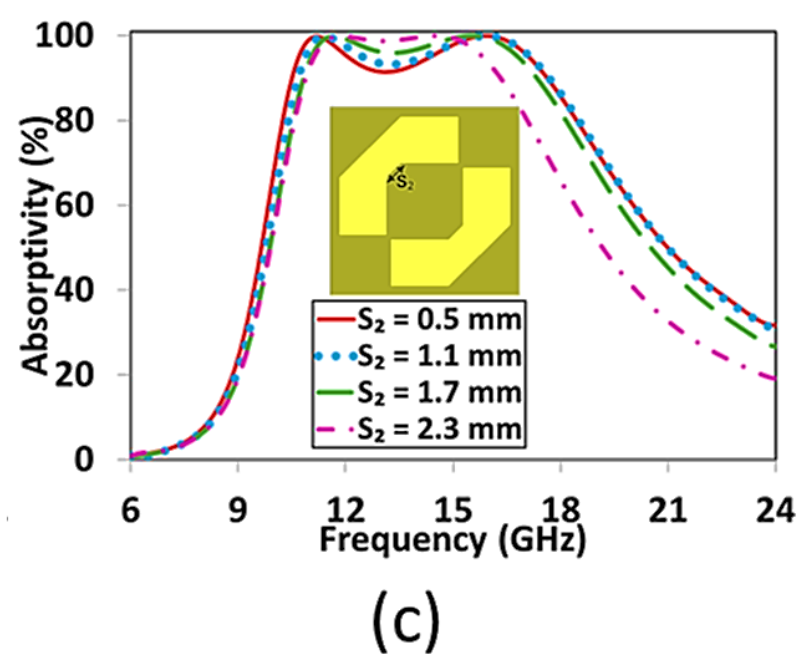

Fig. 8. Simulated absorptivity (a) for different design configurations (b) for different lengths of outer slanting edge (' $\mathrm{S}_{1}$ ') and (c) for different lengths of inner slanting edge (' $\mathrm{S}_{2}$ ').

As ' $\mathrm{S}_{1}$ ' increases to $2.6 \mathrm{~mm}$ the absorption peaks are noticed at 11.13 and $15.99 \mathrm{GHz}$ with $90 \%$ absorption bandwidth of $7.19 \mathrm{GHz}$. For further increase in ' $\mathrm{S}_{1}$ ' to $3.2 \mathrm{~mm}$, the two absorption peaks $\left(f_{1}\right.$ is at $11.31 \mathrm{GHz}$ and $f_{2}$ is at $\left.17.34 \mathrm{GHz}\right)$ gets widely separated and structure provides above $80 \%$ 
absorption bandwidth of $9.89 \mathrm{GHz}$ from 10.38 to $20.27 \mathrm{GHz}$. On the other hand it is observed from Fig. 8 (c) that as ' $\mathrm{S}_{2}$ ' has been increased keeping ' $\mathrm{S}_{1}$ ' fixed the $90 \%$ absorption bandwidth decreases as both the absorption peaks $\left(f_{1} \& f_{2}\right)$ start coming closer. The values of absorption peaks $\left(f_{1} \& f_{2}\right)$ for different dimensions of ' $\mathrm{S}_{2}$ ' are listed in Table II. At ' $\mathrm{S}_{2}$ ' equals to optimized value of $0.5 \mathrm{~mm}$ the two absorption peaks $f_{1}$ and $f_{2}$ are observed at 11.13 and $15.99 \mathrm{GHz}$ respectively, but as ' $\mathrm{S}_{2}$ ' increases to $2.3 \mathrm{~mm}$ absorption peaks are observed at 11.85 and $14.61 \mathrm{GHz}$ with more than $90 \%$ absorption bandwidth of only $5.42 \mathrm{GHz}$ from 10.89 to $16.31 \mathrm{GHz}$.

TABLE II. VARIATIONS IN ABSORPTION FREQUENCIES W.R.T VARIATION IN LENGTH 'S $\mathrm{S}_{2}$.

\begin{tabular}{|c|c|c|c|c|}
\hline S. No. & $\mathbf{S}_{\mathbf{2}}(\mathbf{m m})$ & $f_{\boldsymbol{1}}(\mathbf{G H z})$ & $f_{2}(\mathbf{G H z})$ & $\begin{array}{c}\text { 10 dB Absorption } \\
\text { Bandwidth (GHz) }\end{array}$ \\
\hline 1 & 0.5 & 11.13 & 15.99 & 7.19 \\
\hline 2 & 1.1 & 11.40 & 15.92 & 7.03 \\
\hline 3 & 1.7 & 11.67 & 15.58 & 6.47 \\
\hline 4 & 2.3 & 11.85 & 14.61 & 5.42 \\
\hline 5 & $\begin{array}{c}\text { \% Change in absorption peak } \\
\text { w.r.t change in } \mathrm{S}_{2}\end{array}$ & $6.46 \%$ & $8.63 \%$ & - \\
\hline
\end{tabular}

The proposed absorber has been compared with previously presented metamaterial absorbers for their $10 \mathrm{~dB}$ absorption bandwidth in Table III. It is observed that however [18] and [25] have wide $10 \mathrm{~dB}$ absorption bandwidth as compared to the proposed design but they have multilayer design configuration with larger thickness and unit cell dimensions which makes them unfit for planar applications. The design presented in [26] have smaller unit cell dimensions with electrically thin thickness but its $10 \mathrm{~dB}$ absorption bandwidth is less as compared to the proposed absorber design. A similar configuration with larger unit cell size and comparatively less absorption bandwidth is also presented in [27]. Further, the absorber design presented in [28] have single layer larger configuration with larger absorption bandwidth but its unit cell size is large as compared to the proposed absorber.

TABLE III. COMPARISON OF THE PROPOSED ABSORBER WITH PREVIOUSLY PRESENTED DESIGNS.

\begin{tabular}{|c|c|c|c|c|c|}
\hline Absorber & $\begin{array}{c}\text { Center } \\
\text { Frequency } \\
(\mathbf{G H z})\end{array}$ & $\begin{array}{c}\text { Unit Cell } \\
\text { Size } \\
(\mathbf{m m})\end{array}$ & Thickness $(\mathbf{m m})$ & $\begin{array}{c}\mathbf{1 0} \text { dB Absorption } \\
\text { Bandwidth }\end{array}$ & $\begin{array}{c}\text { Design } \\
\text { Configurations }\end{array}$ \\
\hline$[18]$ & 14.69 & 6.4 & $3.65\left(0.179 \lambda_{0}\right)$ & $85.9 \%$ & Multi-layer \\
\hline$[20]$ & 11.51 & 9.0 & $1.6\left(0.061 \lambda_{0}\right)$ & $46.2 \%$ & Dual Layer \\
\hline$[21]$ & 10.15 & 13.8 & $5.6\left(0.189 \lambda_{0}\right)$ & $45.0 \%$ & Dual Layer \\
\hline$[24]$ & 10.05 & 7.1 & $2.0\left(0.067 \lambda_{0}\right)$ & $43.7 \%$ & Single Layer \\
\hline$[25]$ & 8.78 & 12.0 & $4\left(0.12 \lambda_{0}\right)$ & $86.6 \%$ & Multi-layer \\
\hline$[26]$ & 10.47 & 4.5 & $1.6\left(0.055 \lambda_{0}\right)$ & $35.8 \%$ & Single Layer \\
\hline$[27]$ & 5.9 & 10.0 & $3.2\left(0.063 \lambda_{0}\right)$ & $44.06 \%$ & Single Layer \\
\hline$[28]$ & 11.01 & 8.0 & $3.2\left(0.118 \lambda_{0}\right)$ & $75.38 \%$ & Single Layer \\
\hline $\begin{array}{l}\text { Proposed } \\
\text { Absorber }\end{array}$ & 14.05 & 5.5 & $1.6\left(0.074 \lambda_{0}\right)$ & $51.2 \%$ & \\
\hline
\end{tabular}


Thus the geometric dimensions greatly affect the absorption bandwidth of the proposed structure. It is noticed that the variation of geometrical parameters changes the current paths thereby effective inductance and capacitance of the structure varies which further changes the location of absorption peaks. It is clearly understood from the field, current distributions and analysis of the essential dimensional parameters that the higher absorption peak $\left(f_{2}\right)$ is mainly provided by the outer slanting edge (' $\mathrm{S}_{1}$ ') and width ('W') of the proposed design, whereas the lower absorption peak $\left(f_{l}\right)$ is largely controlled by the length (' $\mathrm{L}_{1}$ '), although the width ('W') and the inner slanting edge (' $\mathrm{S}_{2}$ ') has a small contribution at this frequency. Moreover, in comparison with previously reported absorbers, the proposed design is a monolayer, low profile (unit cell size is $\lambda_{0} / 4$ ) absorber with its ultrathin thickness $\left(0.074 \lambda_{0}\right)$ and gives wideband response with a $10 \mathrm{~dB}$ absorption bandwidth of $7.19 \mathrm{GHz}$, i.e. $51.2 \%$ at the center frequency of $14.05 \mathrm{GHz}$.

\section{EXPERIMENTAL MEASUREMENTS}

For experimental verification a prototype consisting of $45 \times 45$ unit cells has been fabricated as shown in Fig. 9 (a) on a $1.6 \mathrm{~mm}$ thick FR-4 substrate using standard printed circuit board technology. An enlarged portion of the fabricated sample is shown in Fig. $9(b)$. Testing of the prototype has been performed as suggested in $[16,24]$. For the measurements of reflection from the fabricated structure two standard gain UWB horn antennas (VSWR < 2) with frequency range 1 to $18 \mathrm{GHz}$ are used. The antennas are connected to an Agilent N5222A vector network analyzer. One antenna is used as transmitting antenna and the other is used for the reception of the reflected wave. For calibration of the measurement setup, initially a copper plate of identical dimensions is placed in front of antennas in free space at a distance at which effect of near field and diffractions are minimized and the reflection from its surface has been measured. Thereafter, copper sheet is replaced by the fabricated prototype and then the reflection is measured. The difference between the two reflected powers provides the actual reflection from the fabricated absorber structure. In Fig. 9 (c) the measured absorptivity for the normal incidence of the wave is compared with the simulated absorptivity for the 90\% absorption bandwidth. From the measured response it is seen that the absorptivity peaks has been occurring at 11.04 and $15.89 \mathrm{GHz}$ with the absorption values of $99.59 \%$ and $99.28 \%$ respectively, with $90 \%$ absorption bandwidth of $6.92 \mathrm{GHz}$ from 10.52 to $17.44 \mathrm{GHz}$. The calculated error in measured and simulated $10 \mathrm{~dB}$ absorption bandwidth is $0.037 \%$. The sources of error in the measured reflection are the nonlinear behavior of substrate used and the fabrication tolerance. 


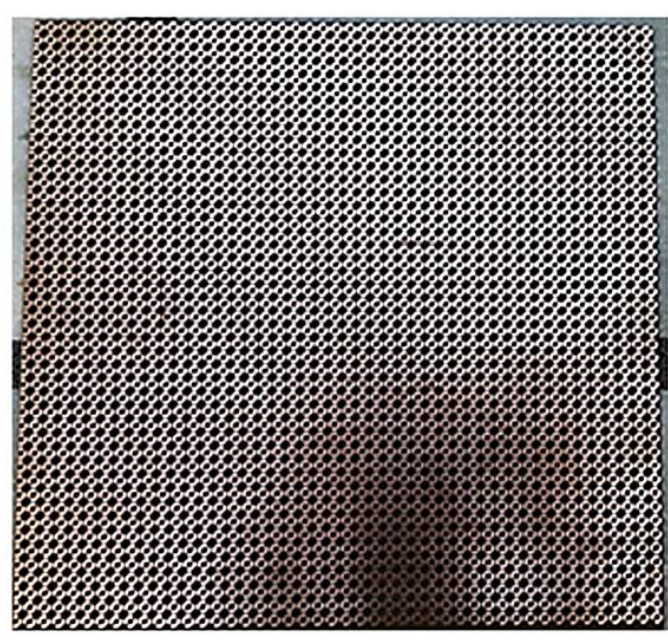

(a)

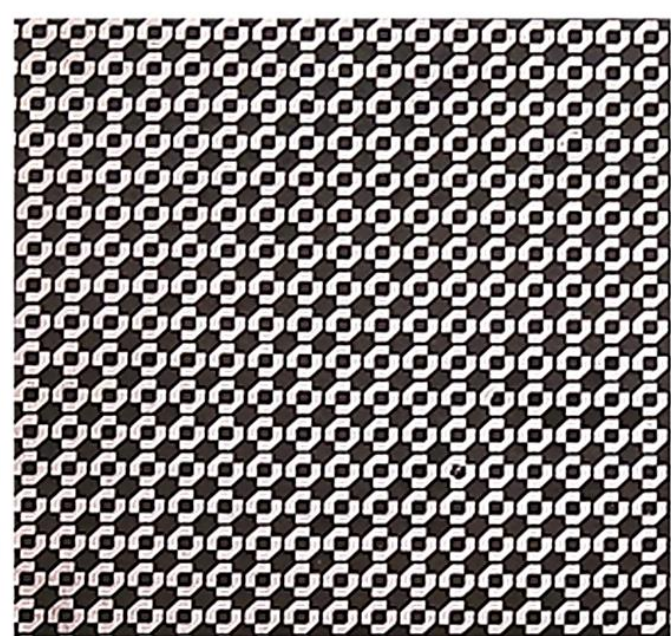

(b)

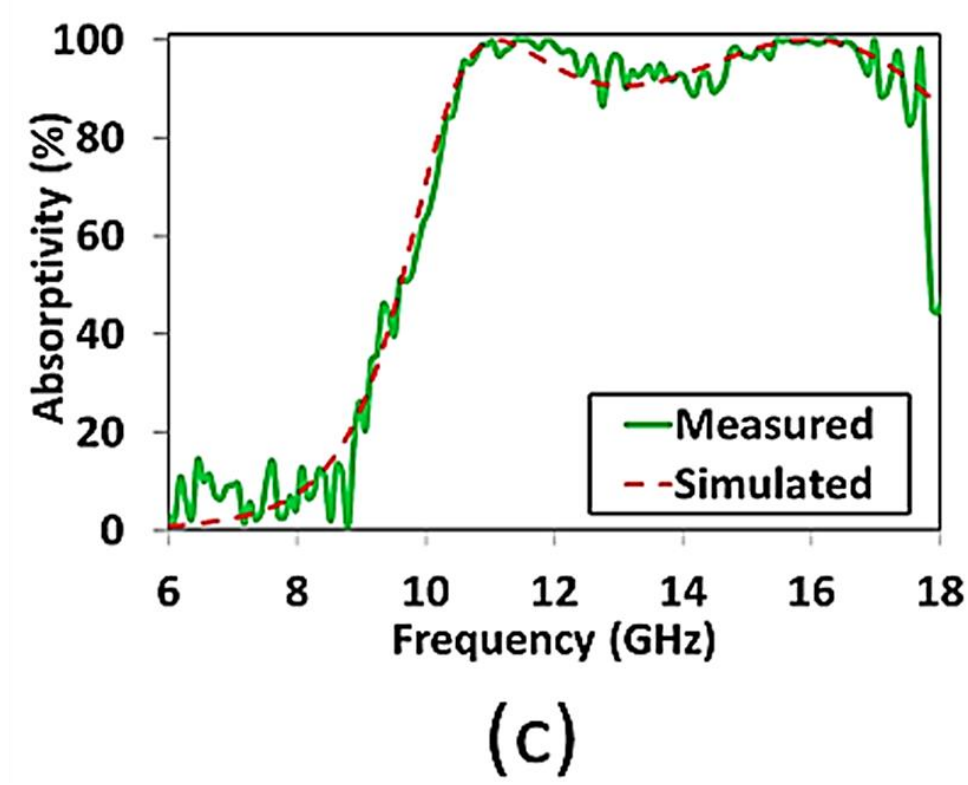

Fig. 9. (a) Fabricated prototype of the absorber (b) its enlarged view and (c) comparison of simulated and measured absorptivity under normal incidence.

The fabricated structure has also been experimentally investigated for different polarization angles (' $\phi$ ') under normal incidence by rotating the structure around its axis from $0^{\circ}$ to $90^{\circ}$ and the measured absorption response is shown in Fig. 10. It is observed that the absorptivity is decreased to minimum as polarization angle increases to $45^{\circ}$ and for further increase in polarization angle absorptivity increases and reaches to maximum absorption at $90^{\circ}$. This measured polarization behavior is in agreement with the simulated response. To investigate the wide angle absorptivity of the proposed absorber, the fabricated structure is experimentally tested for different oblique angles of incidence wave from $0^{\circ}$ to $60^{\circ}$ under both TE and TM polarization as shown in Fig. $11(a)$ and (b). For oblique angle measurements the horn antennas are rotated along the circumference of a circle at the center of which the fabricated structure is placed and the radius of the circle is equal to the distance at which 
near field effects are negligible. For TE polarization as shown in Fig. $11(a)$ it is observed that at the incident angle of $60^{\circ}$ the absorptivity is more than $50 \%$ with the absorption bandwidth of $4.93 \mathrm{GHz}$ from 10.44 to $15.37 \mathrm{GHz}$ which very close to the simulated value of $5.09 \mathrm{GHz}$. Further, for TM polarization as shown in Fig. 11(b) the absorption is above $50 \%$ for a bandwidth of $4.79 \mathrm{GHz}$ from 10.52 to $15.31 \mathrm{GHz}$. Therefore, for higher incidence angles the fabricated absorber shows wideband absorption, which is in agreement with the simulated response.

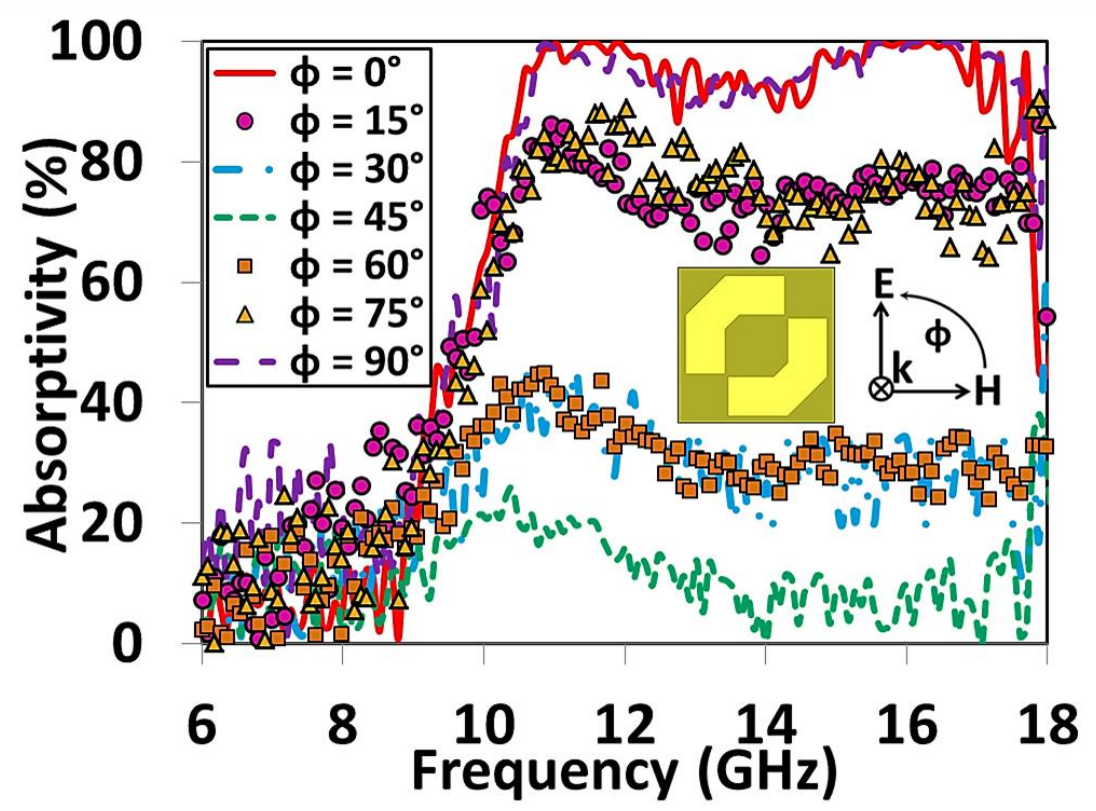

Fig. 10. Measured absorptivity of the proposed absorber (a) for various polarization angles $(\varphi)$ under normal incidence.

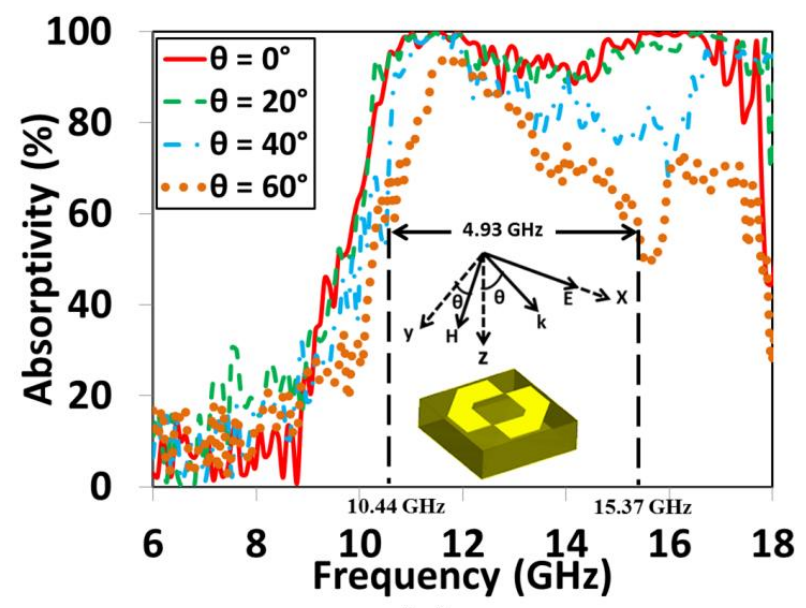

(a)

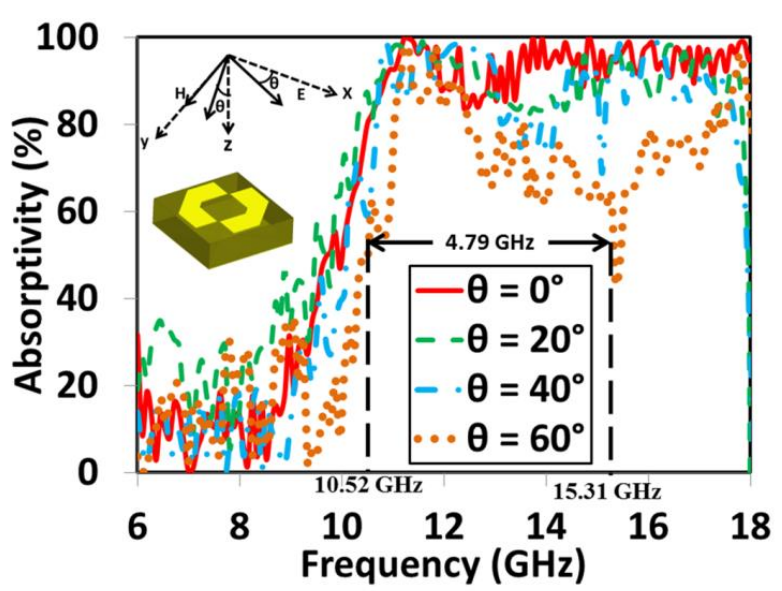

(b)

Fig. 11. Measured absorptivity of the proposed absorber for different oblique incidence angles ( $\theta$ ) for (a) TE and (b) TM polarization. 


\section{CONCLUSIONS}

A novel ultra-wideband ultrathin $\left(\sim \lambda_{0} / 14\right.$ thick corresponding to center frequency), low profile (unit cell size is $\sim \lambda_{0} / 4$ ) and wide-angle metamatetial based microwave absorber has been presented with two diagonally placed 'microstrip bend' shape like patches. The simulation response under normal incidence shows more than $90 \%$ absorption in the frequency range from 10.45 to $17.64 \mathrm{GHz}$ and the FWHM absorption bandwidth is 11.43 $\mathrm{GHz}$ from 9.61 to $21.04 \mathrm{GHz}$. The mechanism of absorption has been analyzed using electromagnetic field and current distributions. The effects of essential dimensional parameters on absorption bandwidth have been studied through parametric optimizations. The absorption response of the proposed absorber under different oblique and polarization angles has been studied. It provides more than $50 \%$ absorption for wideband even for incident angle of $60^{\circ}$. A prototype of the proposed design has been fabricated and tested experimentally. The experimental results are in agreement with the simulated results. It is observed that in comparison to already proposed metamaterial absorbers the proposed design is low profile, ultrathin and has wider $90 \%$ absorption bandwidth. Therefore, the proposed absorber is suitable for various potential applications such as stealth technology, radomes, phase imaging and electromagnetic interference.

\section{ACKNOWLEDGMENT}

This work was financially supported by word bank assisted project TEQIP-II (subcomponent 1.1) under section 12B \& $2 \mathrm{f}$ for graduate studies. The authors want to thank Mr. Saptarshi Ghosh $(\mathrm{PhD}$ Scholar), department of Electrical Engineering, IIT, Kanpur, India for his valuable suggestions for the completion of this work specially measurements and testing.

\section{REFERENCES}

[1] D.R. Smith, W.J. Padilla, D.C. Vier, S.C. Nemat-Nasser, S. Schultz,: "Composite medium with simultaneously negative permeability and permittivity," Phys. Rev. Lett., vol. 84, pp. 4184-4187,2000.

[2] W.J. Padilla, X. Liu, "Perfect electromagnetic absorbers from microwave to optical,”. In SPIE Newsroom, Oct.14, 2010.

[3] T. Liu, X. Cao, J. Gao, Q. Zheng, W. Li, H. Yang, " RCS reduction of waveguide slot antenna with metamaterial absorber," IEEE Trans. Antennas Propag., vol. 61, pp. 1479-1484, 2013.

[4] A. Noor, Z. Hu, "Metamaterial dual polarised resistive Hilbert curve array radar absorber," IET Microwave Antennas Propag. vol. 4, pp. 667-673, 2010.

[5] J. Hao, J. Wang, X. Liu, W.J. Padilla, L. Zhou, M. Qiu, "High performance optical absorber based on a plasmonic metamaterial," App Phys. Lett., vol. 96, pp. 251104, 2010.

[6] J. Rosenberg, R.V. Shenoi, S. Krishna, T.E.Vandervelde, O. Painter, "Design of plasmonic photonic crystal resonant cavities for polarization sensitive infrared photodetectors,” Appl. Phys. Lett., vol. 95, pp. 161101, 2009.

[7] F. Costa, S. Genovesi, A. Monorchio, “A chipless RFID based on multi-resonant high-impedance surfaces," IEEE Trans. Microwave Theory Tech., vol. 61, pp. 146-153, 2013.

[8] D. Schurig, J.J. Mock, B. J. Justice, S.A. Cummer, J.B. Pendry, A.F. Starr, D.R. Smith, "Metamaterial electromagnetic cloak at microwave frequencies," Science, vol. 314, pp. 977-980, 2006.

[9] S. Enoch, G. Tayeb, P. Vincent, “A metamaterial for directive emission,” Phys. Rev. Lett., vol. 89, pp. 3901-3904, 2002.

[10] N. Fang, H. Lee, C. Sun, X. Zhang, "Sub-diffraction limited optical imaging with a silver Superlens," Science, vol. 308, pp. 534-537, 2005. 
[11] H.T. Chen, W. J. Padilla, M. J. Cich, A. K. Azad, R. D. Averitt, A. J. Taylor, “A metamaterial solid state terahertz phase modulator," Nature Photon, vol. 3, pp. 148-151, 2009.

[12] N.I. Landy, S. Sajuyigbe, J. J. Mock, D. R. Smith, W. J. Padilla, "Perfect metamaterial absorber," Phys. Rev. Lett., vol. 100, pp. 207402, 2008.

[13] Q.Y. Wen, H. W. Zhang, Y. S. Xie, Q. H. Yang, Y. L. Liu, "Dual band terahertz metamaterial absorber: design, fabrication, and characterization," Appl. Phys. Lett., vol. 95, pp. 241111, 2009.

[14] H. Tao, C. M. Bingham, D. Pilon, K. Fan, A. C. Strikwerda, D. Shrekenhamer, W. J. Padilla, X. Zhang, R. D. Averitt, “A dual band terahertz metamaterial absorber," J. Phys. D: Appl. Phys. Vol. 43 pp. 225102, 2010.

[15] J. Lee, S. Lim, "Bandwidth-enhanced polarization insensitive microwave metamaterial absorber using double resonance," Electron. Lett., vol. 47,pp. 8-9, 2011.

[16] S. Ghosh, S. Bhattacharyya, Y. Kaiprath, K. V. Srivastava, "Bandwidth enhanced polarization insensitive microwave metamaterial absorber and its equivalent circuit model," J. Appl. Phys., vol. 115,pp. 104503, 2014.

[17] S. Bhattacharyya, S.Ghosh, D. Chaurasiya, K. V. Srivastava, "Bandwidth-enhanced dual-band dual-layer polarizationindependent ultra-thin metamaterial absorber," Appl. Phys. A , vol. 118, pp. 207-215, 2014.

[18] H. Xiong, J. S. Hong, C. M. Luo, L. Zhong, "An ultrathin and broadband metamaterial absorber using multi-layer structures," J. Appl. Phys.,vol. 114, pp. 064109, 2013.

[19] D. Wen, H. Yang, Q. Ye, M. Li, L. Guo, J. Zhang, "Broadband metamaterial absorber based on a multi-layer structure," Phys. Scr., vol. 88, pp. 015402, 2013.

[20] B. Y. Wang, S. B. Liu, B. R. Bian, Z. W. Mao, X. C. Liu, B. Ma, L. Chen, "A novel ultrathin and broadband microwave metamaterial absorber," J. Appl. Phys., vol. 116, pp. 094504, 2014.

[21] S. Bhattacharyya, S. Ghosh, K. V. Srivastava, "Triple band polarization-independent metamaterial absorber with bandwidth enhancement at X-band," J. Appl. Phys., vol. 114, pp. 094514, 2013.

[22] S. Bhattacharyya, S. Ghosh, K. V. Srivastava, "Bandwidth- enhanced metamaterial absorber using electric field driven LC resonator for airborne radar applications," Microw. Opt. Techno. Lett., vol. 55, pp. 2131-2137, 2013.

[23] M. Yoo, S. Lim, "Polarization-independent and ultrawideband metamaterial absorber using a hexagonal artificial impedance surface and a resistor-capacitor layer," IEEE Trans. Antennas Propaga., vol. 62, pp. 2652-2658, 2014.

[24] S. Ghosh, S. Bhattacharyya, D. Chaurasiya, K.V. Srivastava, "An ultrawideband ultrathin metamaterial absorber based on circular split rings,” IEEE Trans. Antennas Propag. Lett., vol. 14, pp. 1172-1175, 2015.

[25] J. Ma, W. Tong, K. Shi, X. Cao, B. Gong, “A Broadband Metamaterial Absorber Using Fractal Tree Structure,” Progress In Electromagnetics Research Lett., vol 49, pp. 73-78, 2014.

[26] D. Sood, C.C. Tripathi, "A wideband ultrathin low profile metamaterial microwave absorber," Microw. Opt. Techno. Lett., 57, pp. 2723-2728, 2015.

[27] S. Bhattacharyya, S. Ghosh, D. Churasiya, K.V. Srivastava, "A Broadband Wide Angle Metamaterial Absorber for Defense Applications," In IEEE International Microw. and RF Conf. (IMARC), Banglore, India, pp. 33-36, 2014.

[28] S. Bhattacharyya, S. Ghosh, D. Churasiya, K.V. Srivastava, "Wide-angle broadband microwave metamaterial absorber with octave bandwidth," IET Microw. Antennas Propaga., vol. 9, pp.1160 - 1166, 2015.

[29] H. X. Xu, G. M. Wang, M. Q. Qi, J. G. Liang, J. Q. Gong, Z. M. Xu, “Triple-Band Polarization Insensitive Wide-Angle Ultra-Miniature Metamaterial Transmission Absorber,” Phys. Rev. B, vol. 86, pp. 205104, 2012.

[30] Y. Pang, H. Cheng, Y. Zhou, J. Wang, “ Analysis and Design of Wire Based Metamaterial Absorbers Using Equivalent Circuit Approach,” J. Appl. Phys., vol. 113, pp. 114902, 2013.

[31] S. Ghosh, K. V. Srivastava, “An Equivalent Circuit Model of FSS Based Metamaterial Absorber Using Coupled Line Theory," IEEE Antennas Wireless Propaga. Lett., vol. 14, pp.511-514, 2015.

[32] F. Costa, S. Genovesi, A. Monorchio, G. Manara, "A circuit based model for the interpretation of perfect metamaterial absorbers," IEEE Trans. Antennas Propaga., vol. 61,pp. 1201-1209, 2013. 